\title{
Sialendoscopy: A Review of 133 Cases
}

\author{
D. S. Deenadayal*, Vyshanavi Bommakanti \\ Department of Otolaryngology and Head and Neck Surgery, Yashoda Hospital, Hyderabad, Secunderabad \\ Email: ${ }^{*}$ aarticlinic@yahoo.com
}

Received 4 December 2015; accepted 24 January 2016; published 27 January 2016

Copyright @ 2016 by authors and Scientific Research Publishing Inc.

This work is licensed under the Creative Commons Attribution International License (CC BY).

http://creativecommons.org/licenses/by/4.0/

(c) () Open Access

\begin{abstract}
Objective: The aim is to review the effectiveness of sialendoscopy in treating various salivary gland disorders related to the duct. Methods: A total of 133 patients who underwent sialendoscopy were included in this study. Data was collected from case records of patients and was analysed. A detailed history examination was taken and the operative findings were analysed. Causes of obstructive sialadenitis were evaluated and treated. Results: A total of 159 glands were subjected to sialendoscopy and pathology was identified in 149 glands. Forty one submandibular glands had calculi and twelve had non-calcular obstruction. Fifteen parotid glands had calculi and 81 had non-calcular obstruction, of which stricture was the most common. Calculi retrieval or dilation of stricture was achieved with a sialendoscope in 112 glands. In 23 patients, the calculi were retrieved by combined approach. In three patients, the calculi were fragmented with intra luminal Holmium laser and the fragments were removed. All these patients had a good relief from their symptoms. Duct perforation was seen in two patients and a false passage was formed in one. Conclusion: Sialendoscopy was a safe and effective procedure for managing ductal pathologies of salivary glands.
\end{abstract}

\section{Keywords}

Sialendoscopy, Calculi, Obstructive Sialadenitis, Mucus Plug, Stricture, Sialolithiasis

\section{Introduction}

Sialendoscopy is a minimally invasive technique that allows diagnosis and treatment of non-neoplastic salivary gland pathology related to the salivary duct. For diagnostic purposes, sialendoscopy has been found to be superior to other modalities of imaging for obstructive pathologies. The most common benign ductal pathology for which sialendoscopy is indicated is salivary stones which account for $60 \%-70 \%$ of salivary duct obstructions. [1]. Majority of stones are found in Wharton's duct followed by Stensons duct [2]. Pediatric sialadenitis ac-

\footnotetext{
"Corresponding author.
} 
counts for $10 \%$ of the salivary gland diseases in children [3]. Most common disorders affecting children are viral parotitis and juvenile recurrent parotitis. Other pathology that affects the ducts is strictures, mucous plugs, polyps, and other ductal abnormalities which cause obstructive sialadenitis [4] [5].

Before the advent of sialendoscopy, these disorders are treated either medically with antibiotics, anti-inflammatory drugs and sialogogues or by surgical excision. While medical treatment doesn't offer permanent cure, surgical excision carries the risk of injury to nerves, mainly the marginal mandibular nerve, lingual nerve, hypoglossal nerve and facial nerve [1]. Surgery is also associated with poor cosmetic outcome, frey's syndrome, paraesthesias, salivary fistulas, wound infections and long recovery time. With the advent of sialendoscopy, there is a shift in the paradigm from surgical to conservative approach.

The first attempt to perform sialendoscopy was by Katz and Gundlach in 1990 [6], but the indications were narrow. With the development of optics and instrumentation, miniaturization of endoscopes, the indications of sialendoscopy have vastly expanded.

Sialendoscopy can be either diagnostic or therapeutic. There are many modalities of diagnosing ductal system pathologies like ultrasound (USG), conventional sialography, computed tomography (CT)—sialography and magnetic resonance (MR)—silography; but each with its own advantages and disadvantages. Sialendoscopy gives endoluminal vision and has a success rate of 96\% - 98\% [7] [8].

The main advantage of sialendoscopy is to preserve the functional gland while relieving the obstruction. It can be performed as a day care procedure with minimal morbidity [9]. It can be performed in any age group and is especially useful in elderly patients who have other associated co-morbidities [10].

Sialendoscopy has few limitatations which include a long learning curve, difficulty in navigating small and delicate ducts with variable branching, need of a trained operator in difficult cases [10].

This article describes the use of sialendoscopy for treating various ductal disorders of the salivary gland.

\section{Materials and Methods}

This is a retrospective study conducted at the department of otolaryngology and head and neck surgery, in a tertiary referral centre. The study is approved by the institute's ethical committee. The duration of study was from October 2010 to June 2015. The data was retrieved from the case records of the patients. On an average 24 cases per year underwent the procedure.

Patients of all age groups were included. A detailed history was noted and those patients who presented with recurrent pain and swelling of the cheek or swelling below the jaw specifically increasing after chewing, fever, or dry mouth were included in this study. A thorough clinical examination was conducted after which they were taken up for sialendoscopy. In the initial part of the study sialendoscopy was performed based on clinical observation alone, but in the latter part patients underwent ultrasound before undergoing sialendoscopy. There was no specific contraindication except that it was not performed in acute sialadenitis.

All procedures were performed in the operation theatre. General anaesthesia was used in pediatric patients and in patients in whom laser fragmentation was done. All the other patients underwent the procedure under local anaesthesia.

Sialendoscopy was performed using the Karl Storz sialendoscopy system as advised by Dr. Marchall. The sialendoscope used for adults has an outer diameter of $1.3 \mathrm{~mm}$ in adults and $0.8 \mathrm{~mm}$ in children. The papilla was identified and then dilated with duct probes and conical dilators. Then the sialendoscope was passed with continuous irrigation and advanced under visualization up to tertiary level of branching. In all patients, antibiotic with steroid were instilled into the gland after the procedure. In patients who had extraction of stone or there was narrowing of the duct, infant feeding tube no 5 was stented after clearing the pathology and left in situ for 1 week. In a few patients in whom there was a large stone or stone adherent to the wall a Holmium laser was used to vaporize the stone or a combined approach was used.

In all patients cause of obstruction was evaluated. They were divided into calculus and non calculus obstructive causes. The site of obstruction was identified and treated accordingly.

\section{Results}

A total of 133 patients with clinical features suggestive of obstructive sialadenitis underwent sialendoscopy and were included in the study. 26 patients underwent bilateral procedures, thus 159 glands were subjected to sialendoscopy. 78 patients were males and 55 patients were females. Sixteen patients were of the paediatric age 
group with the youngest child being 3 years of age. The oldest adult who underwent sialendoscopy was 76 years old.

In the initial part of study, the patients were subjected to sialendoscopy on the basis of clinical features. But in the later part the patients underwent ultrasound before sialendoscopy. A total of 54 glands were evaluated by ultrasound. Ultrasound detected pathology in 27 patients, of which twenty two patients had calculi, one patient had sialectasia, and in four patients the findings were suggestive of Sjogren's disease. In all these 27 patients, the pathology was confirmed by sialendoscopy. The remaining 27 patients, who were negative by USG, sialendoscopy was able to identify the pathology in 25 patients. Twenty three patients had strictures, one patient had a calculus and one patient had a mucus plug. Thus pathology was missed in $50 \%$ of patients by ultrasound. Thus, even though ultrasound is a good modality with high sensitivity for calculi, in non-calculus obstructions, it has low sensitivity. Sialendoscopy had a high sensitivity for identifying non calculus obstruction compared to ultrasound.

A total of 159 glands were evaluated with sialendoscopy. Pathology was identified in 149 patients. In remaining 10 patients no pathology was identified. These patients were discharged with medical treatment and to review if the symptoms recurred. Hence the sensitivity of identifying pathology was 93\% (Table 1).

A total of 53 submandibular glands were evaluated. Calculi were identified in 41 glands ( 3 glands had multiple calculi), stricture was found in five patients, sialectasia in one gland, mucus plug in three glands. In one gland, there was a kink of Wharton's duct at the level of mylohyoid. In two patients, the ductal system was filled with pus, suggestive of an abscess, which was drained. In 21 glands the calculi were floating, in 13 glands they were impacted and in seven glands they were either hilar or intraparenchymal (Table 2).

A total of 96 parotid glands were evaluated. Calculi were identified in 15 glands. Two glands had multiple calculi. Among these fifteen, five glands had floating calculi and ten had impacted calculi. The majority of patients with obstructive parotitis had strictures of the Stenson's duct. Out of these, 70 patients had strictures, 10 patients had mucus plugs and one patient had an abscess. Thus strictures were more common in parotid glands and calculi were more common in submandibular glands (Table 2).

Calculi were present in 56 glands of which 41 stones were present in the submandibular gland and 15 in the

Table 1. Diagnostic sensitivity of sialendoscopy.

Total number of patients

Number of glands in whom sialendoscopy was done

Normal

Pathology
133

159 [26 pts bilateral sialendoscopy was done]

10

149

$149 / 159 * 100=93.71 \%$

Table 2. Pathology in submandibular and parotid glands.

\begin{tabular}{|c|c|c|}
\hline & Submandibular & Parotid \\
\hline No of glands & 53 & 96 \\
\hline Calculi & $\begin{array}{c}41 \\
\text { Floating-21 } \\
\text { Fixed-13 } \\
\text { Hilar/intraglandular-7 } \\
\text { Multiple-3 }\end{array}$ & $\begin{array}{c}15 \\
\text { Floating-5 } \\
\text { Fixed-10 } \\
\text { Multiple-2 }\end{array}$ \\
\hline Stricture & 5 & 70 \\
\hline Mucus plug & 3 & 10 \\
\hline Dilatation & 1 & 0 \\
\hline Others & $\begin{array}{c}3 \\
\text { [1-kink } \\
\text { 2-abscess] }\end{array}$ & 1 \\
\hline Abandoned & 0 & 9 \\
\hline
\end{tabular}


parotid gland. Strictures were identified in 75 glands, of which five were in the submandibular gland, and 70 in the parotid (Table 2).

Calculi retrieval or dilation of strictures was achieved with the sialendoscope alone in 112 glands. In 23 patients (13 submandibular and 10 parotid), the calculi were retrieved by a combined approach. In three patients, 2 submandibular and 1 parotid), the calculi were fragmented with intra luminal Holmium laser and the fragments were removed. In eight parotid glands, a thick stricture was encountered which could not be dilated with a sialendoscope. A false passage was created in one patient. In these nine patients the procedure was abandoned.

Eleven patients with submandibular gland pathology underwent gland excision. Seven patients had hilar or intraparenchymal calculi. One patient had a thick stricture and one patient had a kink in the Wharton's duct at the level of mylohyoid, which could not be dilated by sialendoscopy. The remaining two patients had abscesses.

Eighteen patients were diagnosed clinically as chronic recurrent parotitis. In these sialendoscopy showed either a stricture or mucus plug was a stricture or a mucus plug. Six patients were diagnosed for Sjogrens syndrome of which four patients had ultrasound evidence suggestive of Sjogrens and in two patients there was a clinical suspicion. Three patients were diagnosed for juvenile recurrent parotitis. All the patients had no recurrence of symptoms after performing sialendoscopy.

\subsection{Clinical Outcome}

Almost all the patients had good relief of symptoms post-operatively except nine patients in whom revision sialendoscopy was done. One patient was re-evaluated with sialendoscopy after 20 days and a missed calculus was found in the Wharton's duct and it was removed. Following the procedure, the patient was relieved of his symptoms. Eight patients had a recurrence of symptoms between eight months and two years after the primary procedure. All of them were advised repeat sialendoscopy. Three patients refused a repeat procedure. The remaining five patients were evaluated with sialendoscopy. In one patient the recurrence of symptoms was due to a calculus and laser fragmentation was done. The remaining four patients had strictures, which were dilated. All five patients had good relief from symptoms after the repeat procedure.

\subsection{Complications}

Two patients had thick strictures. Ductal perforation occurred during attempted dilation and was managed conservatively. In one patient a false passage was created during the procedure. No other complications were encountered.

\section{Discussion}

Sialadenitis refers to inflammation of the salivary glands. Obstructive sialadenitis is most commonly due to a calculus (sialolithiasis). Obstructive sialolithiasis can also be caused by stenosis, stricture of ducts or by mucus plug impaction within the ducts. Calculi are most commonly seen in the submandibular gland followed by the parotid gland [2]. In our study, we observed that obstructive parotitis was predominantly caused by strictures of ducts. Strictures, as a cause of obstructive sialadenitis are often unrecognized. Ngu et al. have reported a review of 1362 sialograms and have found strictures to be the cause of $25 \%$ of cases of benign salivary obstruction and commonly affect parotid ducts. They concluded that, strictures as a cause of obstructive sialadenitis is often unrecognized [11]. This leads to significant delay in treatment and a considerable morbidity.

Prior to the advent of sialendoscopy, ultrasound and conventional sialography were used in the diagnosis of non neoplastic disorders of the salivary glands. Conventional sialography is a invasive procedure and carries risk of exposure to radiation, contrast reactions. There is often difficulty in cannulating the ducts.

USG is a diagnostic method that aids in diagnosing non opaque calculi. Stones smaller than $3 \mathrm{~mm}$ may not produce any acoustic shadow and may be missed [12]. There is high inter-observer variability in identifying the pathology. In our series, USG had a good sensitivity of $95.6 \%$ for detecting calculi but a poor sensitivity of $17.8 \%$ for detecting non-calculus cause of obstruction like strictures and mucus plugs. All these investigations are purely diagnostic in nature. Sialendoscopy overcomes this disadvantage as both diagnostic and therapeutic interventions can be performed simultaneously.

Sialendoscopy is mainly used for sialolithiasis but its role in other ductal pathologies is also increasingly being recognized. In our series, in ten patients there were mucus plugs and in seventy two patients there were 
strictures. In three patients pus was seen. Dilatation was observed in one patient.

Sialendoscopy for removal of sialolithiasis has a high success rate. Submandibular calculi were present in 41 glands and 15 parotid glands. All the stones which were $<4 \mathrm{~mm}$ in diameter were successfully removed with sialendoscope alone. Stones, $4 \mathrm{~mm}$ to $8 \mathrm{~mm}$ in diameter can be fragmented with laser and the fragments removed. Laser assisted stone removal was done in three of our patients in the later part of the series. Stones larger than $8 \mathrm{~mm}$ and impacted in the duct were removed by a combined approach. Twenty three patients required the combined approach. Stones impacted in hilum or intraparenchymal stones require gland excision. Only three patients had recurrence of calculi in whom a repeat procedure relieved the symptoms. Hence the success rate of sialendoscopy for sialolithiasis is $94.64 \%$ which is concordant with other studies [13].

As discussed above, sialendoscopy has a higher sensitivity in diagnosis of strictures. Strictures were dilated using balloon. A total of 75 glands had strictures in our series. We could successfully dilate the strictures in 66 glands. In 8 glands the strictures were tight and we couldn't dilate them. In one patient, a false passage was created during dilation of stricture. Except for these nine patients, for whom our interventions were unsuccessful, all other patients had significant improvement. The reported procedural success (resolution of obstruction) reported in the literature ranges from $82 \%$ - 87\% [14] [15].

We observed that patients with chronic recurrent parotitis and juvenile recurrent parotitis had good relief of symptoms after performing sialendoscopy and it can be recommended as a treatment option for children, with no side effects. This has been further supported by a study conducted by Mikolajczak S. et al. [16].

Gland excision was done in 11 [7\%] patients in whom the submandibular gland was excised. The gland preservation rate was 93\%. Similar results were seen in another study [17].

Sialendoscopy is a safe procedure. The incidence of various complications like facial nerve palsy, hypoglossal palsy, haemorrhage, ductal wall injury reported in the literature is low. In our series two patients had ductal perforations, which were managed conservatively and in one patient a false passage was created. In all the three patients the cause was thick stricture of the duct. In a study conducted by Marshal et al. in a series of 450 sialendoscopies they did not report any of the above complications [12]. In a large series of 1000 sialendoscopies, impaction of the grasping forceps was reported in two patients and perforation in three cases were encountered. No other major complications were reported [18]. This is similar to our experience, confirming the safety of sialendoscopy.

Limitations of this procedure include, Hilar stones or impacted stones which cannot be removed with sialendoscope.

A technical limitation include fibrosed duct where there is difficulty to navigate in the duct.

\section{Conclusion}

Sialendoscopy is a safe and effective procedure when performed by trained personnel. It has no major risks and the complications are minimal. It can be used as a diagnostic and a therapeutic tool as it offers an advantage of treating the pathology at the time of diagnosis. It is a minimally invasive procedure that is gaining popularity.

\section{References}

[1] Singh, P.P., Gupta, N., Goyal, A. and Tomar, S. (2012) Interventional Sialendoscopy for Parotid Ductal Calculi: Our Preliminary Experience. Indian Journal of Otolaryngology and Head \& Neck Surgery, 64, 252-256. http://dx.doi.org/10.1007/s12070-011-0361-0

[2] Harrison, J.D. (2009) Causes, Natural History, and Incidence of Salivary Stones and Obstructions. Otolaryngologic Clinics of North America, 42, 927-947. http://dx.doi.org/10.1016/j.otc.2009.08.012

[3] Francis, C.L. and Larsen, C.G. (2014) Pediatric Sialadenitis. Otolaryngologic Clinics of North America, 47, $763-778$. http://dx.doi.org/10.1016/j.otc.2014.06.009

[4] Nahlieli, O. and Baruchin, A.M. (2000) Long-Term Experience with Endoscopic Diagnosis and Treatment of Salivary Gland Inflammatory Diseases. Laryngoscope, 110, 988-993. http://dx.doi.org/10.1097/00005537-200006000-00020

[5] Nahlieli, O., Shacham, R., Yoffe, B. and Eliav, E. (2001) Diagnosis and Treatment of Strictures and Kinks in Salivary Gland Ducts. Journal of Oral and Maxillofacial Surgery, 59, 484-490. http://dx.doi.org/10.1053/joms.2001.22667

[6] Katz, P. (1991) [Endoscopy of the Salivary Glands]. Ann Radiol (Paris), 34, 110-113. http://www.ncbi.nlm.nih.gov/pubmed/1897843

[7] Marchal, F., Dulguerov, P., Becker, M., Barki, G., Disant, F. and Lehmann, W. (2001) Specificity of Parotid Sialen- 
doscopy. Laryngoscope, 111, 264-271. http://dx.doi.org/10.1097/00005537-200102000-00015

[8] Nahlieli, O. and Baruchin, A.M. (1997) Sialoendoscopy: Three Years' Experience as a Diagnostic and Treatment Modality. Journal of Oral and Maxillofacial Surgery, 55, 912-918. http://www.ncbi.nlm.nih.gov/pubmed/9294498 http://dx.doi.org/10.1016/S0278-2391(97)90056-2

[9] Faure, F., Boem, A., Taffin, C., Badot, F., Disant, F. and Marchal, F. (2005) Sialendoscopie diagnostique et interventionnelle. Revue de Stomatologie et de Chirurgie Maxillo-Faciale, 106, 250-252.

http://www.ncbi.nlm.nih.gov/pubmed/16299446

http://dx.doi.org/10.1016/S0035-1768(05)85854-1

[10] Al-Abri, R. and Marchal, F. (2010) New Era of Endoscopic Approach for Sialolithiasis: Sialendoscopy. Sultan Qaboos University Medical Journal, 10, 382-387.

http://www.pubmedcentral.nih.gov/articlerender.fcgi?artid=3074741\&tool=pmcentrez\&rendertype=abstract

[11] Ngu, R.K., Brown, J.E., Whaites, E.J., Drage, N.A., Ng, S.Y. and Makdissi, J. (2007) Salivary Duct Strictures: Nature and Incidence in Benign Salivary Obstruction. Dentomaxillofacial Radiology, 36, 63-67. http://dx.doi.org/10.1259/dmfr/24118767

[12] Marchal, F. and Dulguerov, P. (2003) Sialolithiasis Management: The State of the Art. Archives of OtolaryngologyHead and Neck Surgery, 129, 951-956. http://dx.doi.org/10.1001/archotol.129.9.951

[13] Zenk, J., Koch, M., Klintworth, N., et al. (2012) Sialendoscopy in the Diagnosis and Treatment of Sialolithiasis: A Study on More than 1000 Patients. Otolaryngology—Head and Neck Surgery, 147, 858-863. http://dx.doi.org/10.1177/0194599812452837

[14] Ziegler, C.M., Steveling, H., Seubert, M. and Mühling, J. (2004) Endoscopy: A Minimally Invasive Procedure for Diagnosis and Treatment of Diseases of the Salivary Glands. Six Years of Practical Experience. British Journal of Oral and Maxillofacial Surgery, 42, 1-7. http://www.ncbi.nlm.nih.gov/pubmed/14706291

[15] Marchal, F., Dulguerov, P., Becker, M., Barki, G., Disant, F., Lehmann, W. (2002) Submandibular Diagnostic and Interventional Sialendoscopy: New Procedure for Ductal Disorders. Annals of Otology, Rhinology \& Laryngology, 111, 27-35. http://www.ncbi.nlm.nih.gov/pubmed/11800367 http://dx.doi.org/10.1177/000348940211100105

[16] Mikolajczak, S., Meyer, M.F., Beutner, D. and Luers, J.C. (2014) Treatment of Chronic Recurrent Juvenile Parotitis Using Sialendoscopy. Acta Oto-Laryngologica, 134, 531-535. http://dx.doi.org/10.3109/00016489.2013.879738

[17] Iro, H., Zenk, J. and Koch, M. (2010) Modern Concepts for the Diagnosis and Therapy of Sialolithiasis. HNO, 58, 211217. http://dx.doi.org/10.1007/s00106-009-2075-0

[18] Marchal, F., Dulguerov, P. and Lehmann, W. (1999) Interventional Sialendoscopy. The New England Journal of Medicine, 341, 1242-1243. http://dx.doi.org/10.1056/NEJM199910143411620 